\title{
OSTEOPROTEGERIN (OPG) AND SOLUBLE RECEPTOR ACTIVATOR OF NUCLEAR FACTOR KAPPA B LIGAND (S-RANKL) IN PATIENTS WITH RHEUMATOID ARTHRITIS
}

\author{
By \\ Ilham Aly Motawa ${ }^{1}$, Mohamed Ibrahim Aref ${ }^{1}$, Salama Sad Abd El-Latif ${ }^{1}$, \\ Abd El-Shafy Ahmed Hassieb ${ }^{2}$, Hany Mohamed Hafez Shrif ${ }^{3}$ and Ahmed \\ Mohamed Saied Al-Shorbagy ${ }^{1}$ \\ ${ }^{1}$ Clinical Pathology Department, ${ }^{2}$ Rheumatology, Physical and Rehabilitation Department, \\ ${ }^{3}$ Radiology Department, Faculty of Medicine, Al-Azhar University \\ *Correspondence author: Ahmed Mohamed Saied Al-Shorbagy, \\ E-mail: m.s.elshorbagy@outlook.com
}

\begin{abstract}
Background: Rheumatoid arthritis (RA) is a chronic inflammatory disease that may result in debilitating joint deformities with destruction of bone and cartilage. Inflammation is still considered the pivotal inducer of both components of joint damage. A mechanism of bone destruction that could be dissociated from inflammation was proposed in which osteoclasts activation play a prominent role in bone resorption. RANKL and its natural decoy receptor, osteoprotegerin (OPG), play key roles in osteoclast activation.

Objective: To evaluate osteoprotegrin (OPG) and soluble RANKL (s-RANKL) level in serum of rheumatoid arthritis patients, and to correlate their levels in serum to disease activity and radiological findings (bone loss).

Patients and methods: Fifty five rheumatoid arthritis patients were included in the study. They were classified according to disease activity into 2 groups: The first group was active RA group, and the second was inactive RA group. In addition, RA group was classified according to bone erosions into 2 groups: The first group was RA group with bone erosions and the second group was RA group without bone erosions. The study included 25 healthy individuals of matched age and sex as a control group. All participants were exposed to complete clinical examination, radiological examination, and full laboratory evaluation including osteoclast activity markers (OPG/ RANKL ratio), inflammation markers (ESR, CRP and DAS-28 score), immunological markers (RF and anti-CCP), biochemical markers (calcium, phosphorus and alkaline phosphatase) and complete blood picture (CBC).

Results: OPG/ RANKL ratio was significantly lower in RA group compared to control group. OPG/RANKL ratio was significantly lower in active RA group compared to inactive RA group. OPG/ RANKL ratio was significantly lower in RA group with bone erosion compared to RA group without bone erosions. OPG/ RANKL ratio showed significant negative correlation with both disease duration (in all RA groups) and inflammatory markers (in some not all RA groups). OPG/ RANKL ratio did not correlate with biochemical markers in all RA groups.

Conclusion: OPG/ RANKL ratio could be used to monitor joint damage (bone erosions) progression in patients with RA. Progression of joint damage (bone erosions) due to osteoclast over activity could, at least in part, be dissociated from inflammation. Therefore, targeting OPG/ RANKL ratio may be effective in preventing bone damage in RA patients.
\end{abstract}

Key words: Rheumatoid arthritis (RA), Osteoprotegrin (OPG), Receptor activator of nuclear factor kappa-B ligand (RANKL), disease activity score of 28 joints (DAS-28). 


\section{INTRODUCTION}

A wide variety of cytokines and other mediators of inflammation are expressed in joints of patients with RA. Among of these cytokines, tumor necrosis factor (TNF) plays a critical role in pathogenesis of RA. TNF induces the secretion of multiple pro-inflammatory cytokines, e.g. IL-1, IL-6, IL-8 and granulocyte macrophage colony stimulating factor, mediators of inflammation, e.g. metalloproteinase, prostaglandins and nitric oxide, and up regulates the expression of adhesion molecules on endothelial cells, therefore enhancing the migration of immune and inflammatory cells into joints (Fadda et al., 2015).

Bone is always and continuously remodeled. The discovery of the key factors involved in bone remodeling has moved bone research into a new era. Most notable of these factors, belong to tumor necrosis factor TNF and TNF receptor family, are receptor activator of nuclear factor Kapp++a-B ligand [RANKL/TNFSF11]. The cognate receptor RANK (TNFSF11A], decoy soluble receptor for RANKL, and osteoprotegerin [OPG/TNFSF11b]. had been observed (Kolahi et al., 2017).

A mechanism of bone destruction that could be dissociated from inflammation was proposed. RANKL, known as osteoclast differentiating factor $[\mathrm{ODF}]$ or osteoprotegerin (OPG) ligand, mediates osteoclast genesis and activates mature osteoclasts through binding to its receptor RANK on osteoclasts. RA macrophages could differentiate into osteoclasts, which is dependent on RANKL. In addition, activated RA synovial fibroblasts were shown to express RANKL.
Osteoprotegerin (OPG), also known as osteoclasts inhibitory factor (OIF), is a soluble decoy receptor which blocks osteoclasts differentiation and activation by neutralizing RANKL. It has been shown that OPG is produced by osteoblasts, dendritic cells and B lymphocyte cells in the inflammed synovium of patients with RA. Thus, OPG seems to play an important role in preventing erosions and osteoprosis in RA (Martinez et al., 2016).

The aim of the present work was to evaluate osteoprotegerin (OPG) and soluble RANKL (s-RANKL) levels in serum of rheumatoid arthritis (RA) patients and to correlate their levels in serum to disease activity and radiological findings [bone loss].

\section{PATIENTS AND METHODS}

This cross-sectional study was carried out at the clinical pathology and rheumatology and rehabilitation departments, Faculty of Medicine, Al Azhar University. Al Azhar Ethical Committee approved the study. All candidates filled written consents and they were allowed to leave the study at any point. Fifty five rheumatoid arthritis patients were included in this study. Patients were diagnosed according to American College of Rheumatology/ European league against rheumatism (EULAR) classification criteria for RA patients. The patients were divided into 2 subgroups:

Group (a) included 25 RA patients without rheumatoid disease activity. They were 8 males and 17 females. Their ages ranged from 30-41 years. 


\section{OSTEOPROTEGERIN (OPG) AND SOLUBLE RECEPTOR ACTIVATOR... ${ }^{1001}$}

Group (b) was 30 RA patients with rheumatoid disease activity. They were 10 males and 20 females. Their ages ranged from 32-41 years.

As regard presence of bone erosion the RA patients were reclassified into 2 groups: The first group included 30 patients with bone erosion (c). The second group included 25 patients without bone erosions (d). There were no significant differences between the 2 groups as regard to age and sex.

In addition, 20 apparently normal volunteers' age and sex matched with patients. They were 7 males and 13 females. Their ages ranged from 31-42 years.

\section{Exclusion criteria:}

- Age $>60$ years.

- Duration of disease $<2$ years.

- Other rheumatic or autoimmune diseases.

- Associated systemic disorders (Neoplastic, metabolic, endocrine, cardiac, liver and kidney diseases) could affect bone metabolism.

\section{All studied individuals were subjected to the following:}

A. Full history taking.

B. Thorough clinical examination including local examination of joints.

C. Radiological investigations: Plain Xray of hands and feet as well as any affected joints (Sharp et al., 1985).

D. Assessment of disease activity. DAS28 was used to assess patients disease activity (Prevoo et al., 1995).

E. Laboratory investigations:
1. Complete blood count (CBC).

2. Erythrocyte sedimentation rate (ESR) (Westergren, 1957).

3. Rheumatoid factor (RF) latex agglutination (Hudson and Hay, 1989).

4. C-reactive protein (CRP) latex agglutination.

5. Anticyclic citrullinated peptide antibodies (Anti-CCP).

6. Estimation of osteoprotegerin (OPG) and soluble receptor activator of nuclear factor Kappa-B ligand (sRANKL) by ELISA technique.

\section{Statistical Analysis:}

Data were analyzed using Statistical package for Social Science (SPSS) version 24. Mean and standard deviation for data, $\mathrm{t}$ - test for comparison between 2 parametrical groups. X2 comparison for nun-parametrical groups. Pearson correlation coefficient was used for multiple comparisons between different variables. Mann-Whitney U test was used when comparing between two means (for abnormal distributed data). A one-way analysis of variance (ANOVA): when comparing between more than two means. Post Hoc test: was used for multiple comparisons between different variables. $P$ value $<0.05$ was considered significant. 
ILHAM ALY MOTAWA et al.,

\begin{tabular}{|c|c|c|}
\hline $\begin{array}{c}\text { p- } \\
\text { value }\end{array}$ & As regard activity & $\begin{array}{c}\text { As regard bone } \\
\text { erosion }\end{array}$ \\
\hline P1 & $\begin{array}{c}\text { Active RA vs inactive RA vs } \\
\text { control }\end{array}$ & $\begin{array}{c}\text { Pts with BE vs pts. } \\
\text { Without BE vs control }\end{array}$ \\
\hline P2 & $\begin{array}{c}\text { Active RA vs inactive } \\
\text { RA }\end{array}$ & $\begin{array}{c}\text { Pts. With BE vs } \\
\text { patients without BE }\end{array}$ \\
\hline P3 & Active RA vs control & $\begin{array}{c}\text { Patient with BE vs } \\
\text { control }\end{array}$ \\
\hline P4 & Inactive RA vs control & $\begin{array}{c}\text { Pts. Without BE vs } \\
\text { control }\end{array}$ \\
\hline
\end{tabular}

\section{RESULTS}

There were statistically significant increases in serum RANKL and OPG and significant decrease in OPG/ RANKL ratio in RA group compared to control group. There were significant increase in ESR and percentage of positive CRP test in RA group compared to control group. There were significant increases in percentage of RF and anti-CCP seropositive cases in RA group compared to control group. There was a significant decrease in hemoglobin level in RA group compared to control group. There were no significant difference between RA group and control group as regard to biochemical markers. (Calcium, phosphorus and alkaline phosphatase) (Table 1). 
OSTEOPROTEGERIN (OPG) AND SOLUBLE RECEPTOR ACTIVATOR... 1003

Table (1): Comparison between RA patients and Control group as regard lab. Data

\begin{tabular}{|c|c|c|c|c|}
\hline \multicolumn{2}{|l|}{ Parameters } & \multirow{2}{*}{$\begin{array}{c}\begin{array}{c}\text { RA patients } \\
(\mathbf{n}=\mathbf{5 5})\end{array} \\
10.03 \pm 1.04\end{array}$} & \multirow{2}{*}{$\begin{array}{c}\begin{array}{c}\text { Control } \\
(\mathbf{n}=\mathbf{2 0})\end{array} \\
12.7 \pm 0.9\end{array}$} & \multirow{2}{*}{$\begin{array}{c}\text { P-value } \\
<0.001\end{array}$} \\
\hline $\mathrm{Hb}(\mathrm{g} / \mathrm{dl})$ & Mean \pm SD & & & \\
\hline 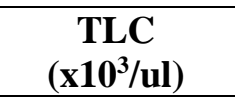 & Mean \pm SD & $6.6 \pm 1.4$ & $6.9 \pm 1.3$ & $>0.05$ \\
\hline $\begin{array}{c}\text { PLT } \\
\left(\mathbf{x 1 0 ^ { 3 } / \mathrm { ul } )}\right)\end{array}$ & Mean \pm SD & $296.2 \pm 60.2$ & $291.3 \pm 55.8$ & $>0.05$ \\
\hline $\mathrm{Ca}(\mathrm{mg} / \mathrm{dl})$ & Mean \pm SD & $8.8 \pm 0.4$ & $8.9 \pm 0.3$ & $>0.05$ \\
\hline Ph (mg/dl) & Mean \pm SD & $3.9 \pm 0.7$ & $3.91 \pm 0.41$ & $>0.05$ \\
\hline $\operatorname{ALP}(\mathbf{U} / \mathbf{L})$ & Mean \pm SD & $201.7 \pm 50.2$ & $222.8 \pm 27.1$ & $>0.05$ \\
\hline ESR (mm/h) & Mean \pm SD & $38.8 \pm 15.7$ & $6.1 \pm 1.6$ & $<0.001$ \\
\hline \multirow{2}{*}{ CRP (mg/L) } & Negative & $28 \quad 50.9 \%$ & $100 \%$ & \multirow{2}{*}{0.002} \\
\hline & Positive & $27 \quad 49.1 \%$ & $0 \%$ & \\
\hline $\begin{array}{c}\text { Anti-CCP } \\
(\mathbf{U} / \mathbf{m l})\end{array}$ & Mean \pm SD & $32 \pm 17.3$ & $4.4 \pm 2.2$ & $<0.001$ \\
\hline \multirow{2}{*}{ Anti-CCP } & Negative & $15 \quad 27.3 \%$ & $20 \quad 100 \%$ & \multirow{2}{*}{$<0.001$} \\
\hline & Positive & $40 \quad 72.7 \%$ & $0 \quad 0 \%$ & \\
\hline \multirow{2}{*}{ RF (iu/ml) } & Negative & $39 \quad 70.9 \%$ & $20 \quad 100 \%$ & \multirow{2}{*}{$<0.025$} \\
\hline & Positive & $16 \quad 29.1 \%$ & $0 \quad 0 \%$ & \\
\hline OPG (ng/ml) & Mean \pm SD & $6.4 \pm 2.3$ & $1.5 \pm 0.3$ & $<0.001$ \\
\hline $\begin{array}{c}\text { RANKL } \\
\text { (ng/ml) }\end{array}$ & Mean \pm SD & $7.7 \pm 1.9$ & $0.8 \pm 0.2$ & $<0.001$ \\
\hline $\begin{array}{c}\text { OPG/ } \\
\text { RANKL } \\
\text { Ratio } \\
\end{array}$ & Mean \pm SD & $0.97 \pm 0.6$ & $1.9 \pm 0.3$ & $<0.001$ \\
\hline
\end{tabular}

There were significant increases in RANKL and significant decrease in both OPG and OPG/ RANKL ratio in active RA group compared to inactive RA group. There was significant increase in ESR, percentage of positive CRP test and DAS28 score in active RA group compared to inactive RA group. There were no significant differences between active RA group and inactive RA group as regard to disease duration, $\mathrm{CBC}$ (hemoglobin, TLC and platelet count), immunological markers (percentage of seropositive cases of RF and anti-CCP) and biochemical markers (calcium, phosphorus and alkaline phosphatase). The level of anti$\mathrm{CCP}$ among seropositive cases was significantly higher in active RA group compared to inactive RA group (Table 2). 
Table (2): Comparison between (active, inactive and control) studied groups as regard laboratory data

\begin{tabular}{|c|c|c|c|c|c|}
\hline \multicolumn{2}{|c|}{$\begin{array}{ll}\text { Parameters } & \text { groups } \\
\end{array}$} & $\begin{array}{c}\text { Active RA } \\
(\mathbf{n}=\mathbf{3 0})\end{array}$ & $\begin{array}{l}\text { Inactive RA } \\
\quad(\mathbf{n}=\mathbf{2 5})\end{array}$ & $\begin{array}{l}\text { Control } \\
(\mathbf{n}=\mathbf{2 0})\end{array}$ & P-value \\
\hline $\mathrm{Hb}(\mathrm{g} / \mathrm{dl})$ & Mean \pm SD & $10.06 \pm 1.09$ & $9.9 \pm 0.9$ & $12.7 \pm 0.9$ & $\begin{array}{l}\mathrm{P} 1<0.001 \\
\mathrm{P} 2=0.795 \\
\mathrm{P} 3<0.001 \\
\mathrm{P} 4<0.001\end{array}$ \\
\hline TLC $\left(\mathrm{x10}^{3} / \mathrm{ul}\right)+$ & Mean \pm SD & $6.6 \pm 1.7$ & $6.6 \pm 1.2$ & $6.9 \pm 1.3$ & $\begin{array}{l}\mathrm{P} 1=0.802 \\
\mathrm{P} 2=0.996 \\
\mathrm{P} 3=0.548 \\
\mathrm{P} 4=0.566\end{array}$ \\
\hline PLT+ $\left(\mathbf{x 1 0}^{3} / \mathbf{u l}\right)$ & Mean \pm SD & $295.7 \pm 61.03$ & $296.8 \pm 60.3$ & $291.3 \pm 55.8$ & $\begin{array}{l}\mathrm{P} 1=0.949 \\
\mathrm{P} 2=0.942 \\
\mathrm{P} 3=0.797 \\
\mathrm{P} 4=0.755\end{array}$ \\
\hline $\mathrm{Ca}(\mathrm{mg} / \mathrm{dl})$ & Mean \pm SD & $8.8 \pm 0.4$ & $8.9 \pm 0.4$ & $8.9 \pm 0.3$ & $\begin{array}{l}\mathrm{P} 1=0.223 \\
\mathrm{P} 2=0.085 \\
\mathrm{P} 3=0.485 \\
\mathrm{P} 4=0.370\end{array}$ \\
\hline Ph (mg/dl) & Mean \pm SD & $3.89 \pm 0.83$ & $3.9 \pm 0.52$ & $3.91 \pm 0.41$ & $\begin{array}{c}\mathrm{P} 1=0.908 \\
\mathrm{P} 2=0.67 \\
\mathrm{P} 3=0.929 \\
\mathrm{P} 4=0.765\end{array}$ \\
\hline $\operatorname{ALP}(\mathbf{U} / \mathbf{L})$ & Mean \pm SD & $207.8 \pm 46.01$ & $201.3 \pm 43.4$ & $222.8 \pm 27.1$ & $\begin{array}{l}\mathrm{P} 1=0.211 \\
\mathrm{P} 2=0.594 \\
\mathrm{P} 3=0.209 \\
\mathrm{P} 4=0.059\end{array}$ \\
\hline $\operatorname{ESR}(\mathbf{m m} / \mathbf{h})$ & Mean \pm SD & $49.1 \pm 13.7$ & $26.4 \pm 5.7$ & $6.1 \pm 1.6$ & $\begin{array}{l}\mathrm{P} 1<0.001 \\
\mathrm{P} 2<0.001 \\
\mathrm{P} 3<0.001 \\
\mathrm{P} 4<0.001\end{array}$ \\
\hline CRP (mg/L) & $\begin{array}{l}\text { Negative } \\
\text { Positive }\end{array}$ & $\begin{array}{cc}3 & 10 \% \\
27 & 90 \% \\
\end{array}$ & $\begin{array}{cl}25 & 100 \% \\
0 & 0 \%\end{array}$ & & $\mathrm{P} 2=0.001$ \\
\hline DAS score & Mean \pm SD & $4.6 \pm 0.9$ & $1.4 \pm 0.5$ & $\begin{array}{ll}----- \\
-\end{array}$ & $<0.001$ \\
\hline $\begin{array}{l}\text { Anti-CCP } \\
\text { (U/ml) }\end{array}$ & Mean \pm SD & $35.7 \pm 16.1$ & $27.5 \pm 18.03$ & $4.4 \pm 2.2$ & $\begin{array}{l}\mathrm{P} 1<0.001 \\
\mathrm{P} 2=0.042 \\
\mathrm{P} 3<0.001 \\
\mathrm{P} 4<0.001\end{array}$ \\
\hline \multirow{2}{*}{ Anti-CCP } & Negative & \begin{tabular}{l|l}
7 & $23.3 \%$
\end{tabular} & $32 \%$ & $100 \%$ & $\begin{array}{l}\mathrm{P} 1<0.001 \\
\mathrm{P} 2=0.472\end{array}$ \\
\hline & Positive & \begin{tabular}{l|l}
23 & $76.7 \%$ \\
\end{tabular} & \begin{tabular}{l|l}
17 & $68 \%$ \\
\end{tabular} & $0 \%$ & $\begin{array}{l}\mathrm{P} 3<0.001 \\
\mathrm{P} 4<0.001\end{array}$ \\
\hline \multirow{2}{*}{ RF (iu/ml) } & Negative & $2170 \%$ & $18 \quad 72 \%$ & & P2 -0431 \\
\hline & Positive & $930 \%$ & $7 \quad 28 \%$ & & $\mathrm{P} 2=0.431$ \\
\hline $\begin{array}{c}\text { Disease Duration } \\
\text { (years) }\end{array}$ & Mean \pm SD & $3.7 \pm 0.79$ & $3.6 \pm 0.96$ & ------ & 0.602 \\
\hline OPG (ng/ml) & Mean \pm SD & $5.7 \pm 2.2$ & $7.3 \pm 2.1$ & $1.5 \pm 0.3$ & $\begin{array}{l}\mathrm{P} 1<0.001 \\
\mathrm{P} 2=0.001 \\
\mathrm{P} 3<0.001 \\
\mathrm{P} 4<0.001\end{array}$ \\
\hline RANKL (ng/ml) & Mean \pm SD & $8.2 \pm 1.7$ & $7.1 \pm 1.9$ & $0.8 \pm 0.2$ & $\begin{array}{l}\mathrm{P} 1<0.001 \\
\mathrm{P} 2=0.008 \\
\mathrm{P} 3<0.001 \\
\mathrm{P} 4<0.001\end{array}$ \\
\hline $\begin{array}{c}\text { OPG/ RANKL } \\
\text { Ratio }\end{array}$ & Mean \pm SD & $0.8 \pm 0.5$ & $1.2 \pm 0.6$ & $1.9 \pm 0.3$ & $\begin{array}{l}\mathrm{P} 1<0.001 \\
\mathrm{P} 2=0.005 \\
\mathrm{P} 3<0.001 \\
\mathrm{P} 4<0.001\end{array}$ \\
\hline
\end{tabular}




\section{OSTEOPROTEGERIN (OPG) AND SOLUBLE RECEPTOR ACTIVATOR... 1005}

There were significant increase in RANKL and significant decrease in both OPG and OPG/ RANKL ratio in RA group with bone erosion compared to RA group without bone erosion. There was significant increase in ESR, percentage of positive CRP test and DAS-28 score in RA group with bone erosion compared to RA group without erosions. There were no significant differences between RA group with bone erosions compared to RA group without bone erosion as regard to CBC (hemoglobin, TLC and platelet count), biochemical markers $(\mathrm{Ca}, \mathrm{ph}$ and alkaline phosphatase) and immunological markers (percentage of seropositive cases of RF and anti-CCP). There was significant increase in disease duration in RA group with bone erosion compared to RA group without bone erosion (Table 3).

Table (3): Comparison between RA patients (without and with bone erosion) as regard laboratory data

\begin{tabular}{|c|c|c|c|c|c|c|}
\hline \multirow{2}{*}{\multicolumn{2}{|c|}{$\mathrm{P}_{\text {Parameters }}$ RA patients }} & \multicolumn{4}{|c|}{ RA patients } & \multirow{3}{*}{$\begin{array}{r}\text { P-value } \\
>0.05\end{array}$} \\
\hline & & \multirow{2}{*}{\multicolumn{2}{|c|}{$\begin{array}{c}\begin{array}{c}\text { Without BE } \\
(\mathbf{n}=\mathbf{2 5})\end{array} \\
10.2 \pm 0.9 \\
\end{array}$}} & \multicolumn{2}{|c|}{$\begin{array}{l}\text { With BE } \\
(\mathbf{n}=\mathbf{3 0})\end{array}$} & \\
\hline Hb (g/dl) & Mean \pm SD & & & & \pm 1.1 & \\
\hline TLC (x10 $3 /$ ul $)$ & Mean \pm SD & \multicolumn{2}{|c|}{$6.4 \pm 1.03$} & & \pm 1.8 & $>0.05$ \\
\hline PLT $\left(\times 10^{3} / \mathrm{ul}\right)$ & Mean \pm SD & \multicolumn{2}{|c|}{$310.1 \pm 60.9$} & \multicolumn{2}{|c|}{$284.6 \pm 57.9$} & $>0.05$ \\
\hline $\mathrm{Ca}(\mathrm{mg} / \mathrm{dl})$ & Mean \pm SD & \multicolumn{2}{|c|}{$8.9 \pm 0.4$} & \multicolumn{2}{|c|}{$8.8 \pm 0.4$} & $>0.05$ \\
\hline $\mathrm{Ph}(\mathrm{mg} / \mathrm{dl})$ & Mean \pm SD & \multicolumn{2}{|c|}{$3.9 \pm 0.7$} & \multicolumn{2}{|c|}{$3.9 \pm 0.7$} & $>0.05$ \\
\hline $\operatorname{ALP}(\mathbf{U} / \mathbf{L})$ & Mean \pm SD & \multicolumn{2}{|c|}{$175.6 \pm 52.3$} & \multicolumn{2}{|c|}{$195.9 \pm 47.3$} & $>0.05$ \\
\hline $\operatorname{ESR}(\mathrm{mm} / \mathrm{h})$ & Mean \pm SD & \multicolumn{2}{|c|}{$28.1 \pm 8.04$} & \multicolumn{2}{|c|}{$47.7 \pm 14.9$} & $<0.001$ \\
\hline \multirow{2}{*}{ CRP (mg/L) } & Negative & 19 & $76 \%$ & 9 & $30 \%$ & \multirow{2}{*}{$<0.001$} \\
\hline & Positive & 6 & $24 \%$ & 21 & $70 \%$ & \\
\hline DAS score & Mean \pm SD & \multicolumn{2}{|c|}{$1.96 \pm 1.14$} & \multicolumn{2}{|c|}{$4.16 \pm 1.6$} & $<0.001$ \\
\hline $\begin{array}{c}\text { Anti-CCP } \\
(\mathbf{U} / \mathrm{ml})\end{array}$ & Mean \pm SD & \multicolumn{2}{|c|}{$20.7 \pm 8.7$} & \multicolumn{2}{|c|}{$41.4 \pm 17.2$} & $<0.001$ \\
\hline \multirow{2}{*}{ Anti-CCP } & Negative & 9 & $36 \%$ & 6 & $20 \%$ & \multirow{2}{*}{$>0.05$} \\
\hline & Positive & 16 & $64 \%$ & 24 & $80 \%$ & \\
\hline \multirow{2}{*}{ RF (iu/ml) } & Negative & 19 & $76 \%$ & 20 & $66.7 \%$ & \multirow{2}{*}{$>0.05$} \\
\hline & Positive & 6 & $24 \%$ & 10 & $33.3 \%$ & \\
\hline $\begin{array}{c}\text { Disease } \\
\text { Duration } \\
\text { (years) }\end{array}$ & Mean \pm SD & \multicolumn{2}{|c|}{$3.66 \pm 0.9$} & \multicolumn{2}{|c|}{$4.2 \pm 0.8$} & 0.044 \\
\hline OPG (ng/ml) & Mean \pm SD & \multicolumn{2}{|c|}{$8.9 \pm 0.6$} & \multicolumn{2}{|c|}{$4.4 \pm 0.4$} & $<0.001$ \\
\hline $\begin{array}{l}\text { RANKL } \\
(\mathrm{ng} / \mathrm{ml})\end{array}$ & Mean \pm SD & \multicolumn{2}{|c|}{$5.8 \pm 0.8$} & \multicolumn{2}{|c|}{$9.3 \pm 0.6$} & $<0.001$ \\
\hline $\begin{array}{c}\text { OPG/ RANKL } \\
\text { Ratio }\end{array}$ & Mean \pm SD & \multicolumn{2}{|c|}{$1.6 \pm 0.3$} & \multicolumn{2}{|c|}{$0.5 \pm 0.05$} & $<0.001$ \\
\hline
\end{tabular}

Correlation study between (OPG, RANKL \& OPG/ RANKL ratio) and other studied parameters in RA patients group.

As regard OPG, there were:

- Statistical significant:
- Positive correlation between OPG \& OPG/ RANKL ratio in RA patients group.

- Negative correlation between (OPG \& DAS score), (OPG \& ESR), (OPG \& Anti-CCP) and (OPG \& RANKL) in RA patients group. 
- Negative correlation between OPG and disease duration in RA group.

- No statistical significant correlation between OPG \& other parameters in RA patients group.

As regard RANKL, there were:

- Statistical significant:

- Positive correlation between (RANKL \& DAS score), (RANKL \& ESR) and (RANKL \& Anti-CCP) in RA patients group.

- Negative correlation between (RANKL \& OPG) and (RANKL \& ratio) in RA patients group.

- Positive correlation between RANKL and disease duration in RA group.
- No statistical significant correlation between RANKL \& other parameters in RA patients group.

As regard OPG/ RANKL Ratio, there were:

- Statistical significant:

- Positive correlation between ratio \& OPG in RA patients group.

- Negative correlation between (Ratio \& DAS score), (Ratio \& ESR), (Ratio \& Anti-CCP) and (Ratio \& RANKL) in RA patients group.

- Negative correlation between Ratio and disease duration in RA group.

- No statistical significant correlation between ratio \& other parameters in RA patients group (Table 4).

Table (4): Correlation study between (OPG, RANKL \& OPG/ RANKL ratio) and other studied parameters in RA patients group

\begin{tabular}{|c|c|c|c|c|c|c|}
\hline \multirow{2}{*}{ RA Patients } & \multicolumn{2}{|c|}{ OPG } & \multicolumn{2}{c|}{ RANKL } & \multicolumn{2}{c|}{$\begin{array}{c}\text { OPG/RANKL } \\
\text { Ratio }\end{array}$} \\
\cline { 2 - 7 } Parameters & $(\mathbf{r})$ & $\mathbf{p}$-value & (r) & p-value & (r) & p-value \\
\hline $\begin{array}{c}\text { Disease } \\
\text { Duration } \\
\text { (Years) }\end{array}$ & -0.31 & 0.02 & 0.33 & 0.014 & -0.43 & 0.001 \\
\hline DAS score & -0.64 & $<0.001$ & 0.56 & $<0.001$ & -0.61 & $<0.001$ \\
\hline $\begin{array}{c}\text { ESR } \\
\text { (mm/h) }\end{array}$ & -0.66 & $<0.001$ & 0.57 & $<0.001$ & -0.63 & $<0.001$ \\
\hline $\begin{array}{c}\text { Anti-CCP } \\
\text { (U/ml) }\end{array}$ & -0.55 & $<0.001$ & 0.62 & $<0.001$ & -0.59 & $<0.001$ \\
\hline $\begin{array}{c}\text { OPG } \\
\text { (ng/ml) }\end{array}$ & -0.92 & $<0.001$ & -0.92 & $<0.001$ & 0.96 & $<0.001$ \\
\hline $\begin{array}{c}\text { RANKL } \\
\text { (ng/ml) }\end{array}$ & $-0 .-$ & ---- & -0.97 & $<0.001$ \\
\hline $\begin{array}{c}\text { OPG/ RANKL } \\
\text { Ratio }\end{array}$ & 0.96 & $<0.001$ & -0.97 & $<0.001$ & & --- \\
\hline
\end{tabular}

(r): Pearson correlation coefficient.

Correlation study between (OPG, RANKL \& OPG/ RANKL ratio) and other studied parameters in active $R A$ group.

As regard OPG, there were:

- Highly statistical significant:

- Positive correlation between OPG \& OPG/RANKL ratio in Active RA group.
- Negative correlation between (OPG \& DAS score), (OPG \& ESR), (OPG \& RANKL) in Active RA group.

- Statistically significant Negative correlation between (OPG \& disease duration) and (OPG and Anti-CCP) in Active RA group. 
- No statistical significant correlation between OPG \& other parameters in Active RA group.

As regard RANKL, there were:

-Highly statistical significant:

- Positive correlation between (RANKL \& DAS score) and (RANKL \& ESR) in Active RA group.

- Negative correlation between (RANKL \& OPG) and (RANKL \& OPG/RANKL ratio) in Active RA group.

- Statistically significant Positive correlation between (RANKL and disease duration) and (RANKL and Anti-CCP) in Active RA group.
- No statistical significant correlation between RANKL \& other parameters in Active RA group.

As regard OPG/RANKL Ratio, there were:

- Highly statistical significant:

- Positive correlation between ratio \& OPG in Active RA group.

- Negative correlation between (Ratio \& DAS score), (Ratio \& ESR), (Ratio \& RANKL) in Active RA group.

- Statistically significant Negative correlation between (ratio and disease duration) and (Ratio and Anti-CCP) in Active RA group.

- No statistical significant correlation between Ratio \& other parameters in Active RA group (Table 5).

Table (5): Correlation study between (OPG, RANKL \& OPG/ RANKL ratio) and other studied parameters in active $\mathrm{RA}$ group

\begin{tabular}{|c|c|c|c|c|c|c|}
\hline \multirow{2}{*}{ Active RA } & \multicolumn{2}{|c|}{ OPG } & \multicolumn{2}{c|}{ RANKL } & \multicolumn{2}{c|}{ OPG/ RANKL Ratio } \\
\cline { 2 - 7 } Parameters & $(\mathbf{r})$ & p-value & $(\mathbf{r})$ & p-value & $(\mathbf{r})$ & p-value \\
\hline $\begin{array}{c}\text { Disease } \\
\text { Duration } \\
\text { (Years) }\end{array}$ & -0.51 & 0.002 & 0.54 & 0.002 & -0.52 & 0.003 \\
\hline DAS score & -0.79 & $<0.001$ & 0.76 & $<0.001$ & -0.81 & $<0.001$ \\
\hline $\begin{array}{c}\text { ESR } \\
\text { (mm/h) }\end{array}$ & -0.77 & $<0.001$ & 0.72 & $<0.001$ & -0.79 & $<0.001$ \\
\hline $\begin{array}{c}\text { Anti-CCP } \\
\text { (U/ml) }\end{array}$ & -0.52 & 0.003 & 0.56 & 0.001 & -0.52 & 0.003 \\
\hline $\begin{array}{c}\text { OPG } \\
\text { (ng/ml) }\end{array}$ & -0.90 & $<0.001$ & -0.90 & $<0.001$ & 0.97 & $<0.001$ \\
\hline $\begin{array}{c}\text { RANKL } \\
\text { (ng/ml) }\end{array}$ & $-0 .-$ & ---- & -0.96 & $<0.001$ \\
\hline $\begin{array}{c}\text { OPG/ } \\
\text { RANKL Ratio }\end{array}$ & 0.97 & $<0.001$ & -0.96 & $<0.001$ & & -- \\
\hline
\end{tabular}

(r): Pearson correlation coefficient.

Correlation study between (OPG, RANKL \& OPG/ RANKL ratio) and other studied parameters in inactive RA group.

As regard OPG, there were:

- Highly statistical significant:
- Positive correlation between OPG \& OPG/ RANKL ratio in inactive RA group.

- Negative correlation between (OPG \& RANKL) in inactive RA group.

- Statistically significant Negative correlation between (OPG \& disease 
duration) and (OPG and Anti-CCP) in inactive RA group.

- No statistical significant correlation between OPG \& other parameters in inactive RA group.

As regard RANKL, there were:

- Highly statistical significant Negative correlation between (RANKL \& OPG) and (RANKL \& OPG/ RANKL ratio) in inactive RA group.

- Statistically significant Positive correlation between (RANKL and disease duration) and (RANKL and Anti-CCP) in inactive RA group.

- No statistical significant correlation between RANKL \& other parameters in inactive RA group.

Table (6): Correlation study between (OPG, RANKL \& OPG/ RANKL ratio) and other studied parameters in inactive $R A$ group

\begin{tabular}{|c|c|c|c|c|c|c|}
\hline \multirow{2}{*}{ Inactive RA } & \multicolumn{2}{|c|}{ OPG } & \multicolumn{2}{c|}{ RANKL } & \multicolumn{2}{c|}{ OPG/ RANKL Ratio } \\
\cline { 2 - 7 } Parameters & $(\mathrm{r})$ & $\mathrm{p}$-value & $(\mathrm{r})$ & $\mathrm{p}$-value & $(\mathrm{r})$ & $\mathrm{p}$-value \\
\hline $\begin{array}{c}\text { Disease Duration } \\
\text { (Years) }\end{array}$ & -0.52 & 0.02 & 0.61 & 0.002 & -0.57 & 0.003 \\
\hline $\begin{array}{c}\text { Anti-CCP } \\
\text { (U/ml) }\end{array}$ & -0.51 & 0.01 & 0.62 & 0.001 & -0.58 & 0.002 \\
\hline $\begin{array}{c}\text { OPG } \\
\text { (ng/ml) }\end{array}$ & -0.93 & $<0.001$ & -0.93 & $<0.001$ & 0.95 & $<0.001$ \\
\hline $\begin{array}{c}\text { RANKL } \\
\text { (ng/ml) }\end{array}$ & 0.95 & $<0.001$ & -0.98 & $<0.001$ & -0.98 & $<0.001$ \\
\hline $\begin{array}{c}\text { OPG/ RANKL } \\
\text { Ratio }\end{array}$ & & --- & --- \\
\hline
\end{tabular}

(r): Pearson correlation coefficient.

Correlation study between (OPG, RANKL \& OPG/ RANLK ratio) and other studied parameters in patients without BE.

As regard OPG, there were:

- Statistically significant:

- Positive correlation between (OPG and Anti-CCP) and (OPG \& OPG/ RANKL ratio) in patients without BE.

- Negative correlation between (OPG and disease duration).
As regard OPG/ RANKL Ratio, there were:

- Highly statistical significant:

- Positive correlation between ratio \& OPG in inactive RA group.

- Negative correlation between (OPG/ RANKL Ratio \& RANKL) in inactive RA group.

- Statistically significant Negative correlation between (OPG/ RANKL Ratio \& disease duration) and (OPG/ RANKL Ratio and Anti-CCP) in inactive RA group.

- No statistical significant correlation between Ratio \& other parameters in inactive RA group (Table 6). 
- Highly statistical significant Negative correlation between ratio \& RANKL in patients without BE.

- Statistically significant Positive correlation between (ratio and OPG) in patients without BE.
- Statistically significant negative correlation between ratio \& disease duration

- No statistical significant correlation between OPG \& other parameters in patients without BE (Table 7).

Table (7): Correlation study between (OPG, RANKL \& OPG/ RANLK ratio) and other studied parameters in patients without $\mathrm{BE}$

\begin{tabular}{|c|c|c|c|c|c|c|}
\hline \multirow{2}{*}{ Pt. without } & \multicolumn{2}{|c|}{ OPG } & \multicolumn{2}{c|}{ RANKL } & \multicolumn{2}{c|}{$\begin{array}{c}\text { OPG/ RANKL } \\
\text { Ratio }\end{array}$} \\
\cline { 2 - 7 } Parameters & $(\mathbf{r})$ & p-value & $(\mathbf{r})$ & p-value & $(\mathbf{r})$ & p-value \\
\hline $\begin{array}{c}\text { Disease } \\
\text { Duration } \\
\text { (Years) }\end{array}$ & -0.64 & 0.001 & 0.51 & 0.009 & -0.58 & 0.002 \\
\hline $\begin{array}{c}\text { Anti-CCP } \\
\text { (U/ml) }\end{array}$ & 0.50 & 0.011 & 0.44 & 0.03 & -0.16 & $>0.05$ \\
\hline $\begin{array}{c}\text { OPG } \\
\text { (ng/ml) }\end{array}$ & & ---- & -0.19 & $>0.05$ & 0.55 & 0.004 \\
\hline $\begin{array}{c}\text { RANKL } \\
\text { (ng/ml) }\end{array}$ & -0.19 & $>0.05$ & & --- & -0.92 & $<0.001$ \\
\hline $\begin{array}{c}\text { OPG/ RANKL } \\
\text { Ratio }\end{array}$ & 0.55 & $0.004 \mathrm{~S}$ & -0.92 & $<0.001$ & & ---- \\
\hline
\end{tabular}

(r): Pearson correlation coefficient.

Correlation study between (OPG, RANKL \& OPG/ RANKL ratio) and other studied parameters in patients with BE.

As regard OPG, there were:

- Highly statistical Positive correlation between (OPG and OPG/RANKL ration) in patients with $\mathrm{BE}$.

- Statistically significant Negative correlation between (OPG and disease duration) in patients with BE.

- No statistical significant correlation between OPG \& other parameters in patients with BE.

As regard RANKL, there were:

- Highly statistical significant Negative correlation between (RANKL \& ratio) in patients with BE.
- Statistically significant Positive correlation between (RANKL and disease duration) in patients with $\mathrm{BE}$.

- No statistical significant correlation between RANKL \& other parameters in patients with $\mathrm{BE}$.

As regard OPG/ RANKL Ratio, there were:

- Highly statistical significant:

- Positive correlation between ratio \& OPG in patients with BE.

- Negative correlation between ratio \& RANKL in patients with BE.

- Statistically significant Negative correlation between (Ratio and disease duration) in patients with $\mathrm{BE}$.

- No statistical significant correlation between OPG \& other parameters in patients with BE (Table 8). 
Table (8): Correlation study between (OPG, RANKL \& OPG/ RANKL ratio) and other studied parameters in patients with $\mathrm{BE}$

\begin{tabular}{|c|c|c|c|c|c|c|}
\hline Pts with BE & \multicolumn{2}{|c|}{ OPG } & \multicolumn{2}{c|}{ RANKL } & \multicolumn{2}{c|}{$\begin{array}{c}\text { OPG/ RANKL } \\
\text { Ratio }\end{array}$} \\
\cline { 2 - 7 } Parameters & $(\mathbf{r})$ & $\mathbf{p}$-value & $(\mathbf{r})$ & $\mathbf{p}$-value & $(\mathbf{r})$ & p-value \\
\hline $\begin{array}{c}\text { Disease Duration } \\
\text { (Years) }\end{array}$ & -0.44 & 0.013 & 0.57 & 0.001 & -0.46 & 0.011 \\
\hline $\begin{array}{c}\text { OPG } \\
\text { (ng/ml) }\end{array}$ & & --- & -0.04 & $>0.05$ & 0.81 & $<0.001$ \\
\hline $\begin{array}{c}\text { RANKL } \\
\text { (ng/ml) }\end{array}$ & -0.04 & $>0.05$ & & ---- & -0.62 & $<0.001$ \\
\hline OPG/ RANKL Ratio & 0.81 & $<0.001$ & -0.62 & $<0.001$ & & --- \\
\hline
\end{tabular}

(r): Pearson correlation coefficient.

\section{DISCUSSION}

Activated T lymphocytes, a proinflammatory cytokine milieu, and their interactions with cells of the bone microenvironment and the immune system cells have been suggested as potential mechanisms that promote the differentiation and activation of osteoclasts and lead to enhanced bone resorption. Receptor activator of nuclear factor $\mathrm{kB}$ ligand (RANKL) is the essential cytokine signal for various osteoclast functions. RANKL is expressed on the membrane of cells of the osteoplastic lineage. Activated $\mathrm{T}$ lymphocytes in addition to expression of membrane RANKL secrete soluble RANKL after being cleaved from membrane bound form by the action of tumor necrosis factor $\alpha$ converting enzyme. The secreted RANKL form has a comparable biological activity as membrane form. Both forms of RANKL act through binding to and activating receptor activator of $\mathrm{NF}-\mathrm{kB}$ (RANK), a cell bound receptor of the TNF receptor (TNFR) superfamily, which is located on osteoclast precursor cells, mature osteoclasts and dendritic cells. The potent stimulatory effects of RANK by RANKL are counterbalanced by an endogenous antagonist called osteoprotegerin (OPG). OPG is secreted as a decoy receptor by many tissues (including cells of immune system) and neutralizes all forms of RANKL through binding to it (Aadhaar et al., 2018).

RANKL-RANK interactions are critical for 2 pivotal functions of the immune system: (1) Early development and maturation of pre-B cells and pre- $\mathrm{T}$ cells in the bone marrow and thymus as they are required for the formation and function of lymphoid structures. (2) They are involved in the interactions between mature DCs and T cells. Thus they have a role in modulating immune responses and preventing inadequate activation of auto reactive $\mathrm{T}$ cells. These effects are antagonized by OPG. The expression of RANK by osteoclasts and DCs, the production of RANKL by osteoplastic lineage cells and activated $\mathrm{T}$ cells and the requirement of RANKL/RANK for the development of normal bone and lymphoid tissues (evident from the phenotypes of knockout mice) all support the concept that the pleiotropic RANKLRANK-OPG system represents a central molecular link between bone metabolism and the immune system. Various 
proinflammatory and proresorptive cytokines (TNF- $\alpha$, IL1, IL6, IL11, IL17 and M-CSF) which initiated by synovitis and enforced by otitis have been detected in inflamed synovial tissues and have been implicated as mediators of bone and cartilage loss through stimulation of osteoclast bone resorption. As RANKLRANK-OPG is essential in bone metabolism and immune function, it was hypothesized that these cytokines converge at the level of RANKL and OPG and that proresorptive cytokines affect bone resorption through modulation of the RANKL: OPG ratio. Explanation seemed to be rational as RA disease is characterized by both inflammation and bone destruction. On the other hand it has been reported that IL1 and TNF- $\alpha$ induce osteoclast activation through a RANKL independent pathway. Thus RANKRANKL-OPG axis and inflammation could, in part, be dissociated. So drugs modulating RANKL-RANK-OPG axis could be potential target for novel therapeutic agents (Sakae, 2019).

From aforementioned reports our target was to evaluate RANKL-RANK-OPG axis in RA exploring their relation to each other and to correlate their levels to disease activity and bone loss.

RF and anti-CCP are involved in 2010 ACR/ EULAR disease classification criteria. In our study among RA group, $29.1 \%$ were RF seropositive and $72.7 \%$ were anti CCP seropositive. Both RF and anti CCP seropositive cases were significantly higher in RA group compared to control group. Neither RF nor anti $\mathrm{CCP}$ as a percentage of seropositive test had the potential to differentiate active RA group from inactive RA group and RA group with bone erosion from RA group without bone erosion. But the levels of anti CCP were significantly higher in active RA group compared to inactive RA group and in RA group with bone erosion compared to RA group without bone erosion. These findings are similar to studies done by Conrad et al. (2010), Syversen et al. (2010) and De-Punder et al (2013) who reported that anti CCP specifically interact with citrullinated vimentin (CV) expressed on the membrane of osteoclast precursors. On the other hand our results are contradictory to study done by Barra et al. (2014) who observed that anti CCP seronegative patients had more severe disease compared to anti-CCP seropositive patients in the terms of disease activity and radiographic damage. These contradictory results could be attributed to the used antirheumatic drugs.

In our study, RA group showed increased level of RANKL and OPG and lowered level of OPG: RANKL ratio compared to control group. These findings are agreed with studies done by Kazim et al., (2015). Sara et al. (2016) and Sousan et al. (2016). Other studies done by $\mathrm{Xu}$ et al. (2012) and Fadda et al. (2015) disagree with our findings as regards OPG levels. They observed increased OPG levels in healthy control group compared to RA group. This discrepancy could probably be attributed to the fact that RANKL and OPG behave differently and are secreted by different cells. So, it has been suggested that OPG: RANKL ratio may be more important than estimation of single OPG or RANKL levels to monitor bone homeostasis in RA patients Fadda et al. (2015). In our study, serum RANKL levels were significantly higher in active 
RA group compared to inactive RA group. Also serum OPG and OPG: RANKL ratio was significantly lower in active RA group compared to inactive RA group. These findings are agree with studies done by Kazim et al. (2015) and Papadaki et al. (2019), but contradictory to studies done by Kolahi et al. (2017). In addition in our study, serum RANKL was significantly higher in RA group with bone erosion compared to RA group without bone erosion and both serum level of OPG and OPG: RANKL ratio were significantly lower in RA group with bone erosion compared to RA group without bone erosion. The duration of disease and the inflammatory markers (ESR, CRP and DAS score) were significantly higher in RA group with bone erosion compared to RA group without bone erosion. These findings agree with studies done by Kazim et al. (2015) and Sakae, (2019), but contradictory to study done by Aadhaar et al. (2018). The differences between studies could be explained by age range, sample size, disease duration, drugs used in treatment, degree of disease activity and degree of disease severity which are not necessary comparable between studies.

In our study, the best relationship between bone homeostasis markers (OPG, RANKL and OPG: RANKL ratio) throughout all the studied groups observed using OPG: RANKL ratio. This ratio showed positive correlation with OPG and negative correlation with RANKL. So we prefer estimation of OPG: RANKL ratio compared to single estimation of OPG or RANKL to monitor bone homeostasis in RA patients. This observation is going with study done by Fadda et al. (2015).
In our study, OPG: RANKL ratio as a measure of osteoclasts over activity and a predictor of bone erosion was correlated with disease duration in all RA studied groups and with inflammatory markers (CRP \& ESR and DAS score) and anti$\mathrm{CCP}$ (that could share in the process of inflammation) in RA group and active RA group but not with other RA groups including RA group with bone erosion. These observations indicate that inflammation and progression of bone erosion can, at least in part, be dissociated and confirmed the clinical point of view that bone erosion can occur in absence of inflammation. These findings are similar to studies done by Fadda et al, (2015), but contradictory to studies done by Van Tuyl et al. (2010) and Gerolamo et al. (2012) who confirmed a positive correlation between bone homeostasis markers (OPG, RANKL and OPG: RANKL ratio) and inflammatory markers. They confirmed the clinical point of view that joint destruction is unlikely to be induced in absence of inflammation but inflammation can occur in absence of bone erosion. This controversy between studies could be attributed to differences in disease duration, disease activity and disease severity. In our study, bone homeostatic markers (OPG, RANKL and OPG: RANKL ratio) did not correlate with bone biomarkers (Calcium, phosphorus and alkaline phosphatase) in all the studied groups of RA patients. So, they cannot be used to reflect RA disease activity or progression in bone erosion. These observations are contradictory to studies done by Sridevi and Vinit (2019). 


\section{RECOMMENDATIONS}

Estimation of serum OPG: RANKL ratio to monitor joint damage progression in patients with RA is needed. Progression of joint damage (bone erosions) due to osteoclast over activity could, at least in part, be dissociated from inflammation. Therefore, targeting the RANK-RANKLOPG system may be effective in preventing bone damage in RA patients.

\section{REFERENCES}

1. Aadhaar D, Narendhiran $P$, Karthik VM, Suchet S, Aman S, Shefali S and Nidhi GV (2018): Circulating levels of osteoprotegerin and sRANKL and the effect of methotrexate in patients with rheumatoid arthritis. Indian Journal of Rheumatology, 13 (2): 90-94.

2. Barra L, Pope J, Orav J, Boire G, Haraoui B, Hitchon C, Keystone E, Thorne J, Tin D and Bykerk V (2014): Prognosis of seronegative patients in a large prospective cohort of patients with early inflammatory arthritis. The Journal of Rheumatology, 41, (12): 2361-2369.

3. Conrad K, Roggenbuck D, Reinhold $D$ and Dorner T (2010): Profiling of rheumatoid arthritis associated autoantibodies. Autoimmune Rev., 9 (6): 431-5.

4. De-Punder Y, Hendrikx J, Den-Broeder A, Valls-Pascual E, Van-Riel $P$ and Fransen $J$ (2013): Should we redefine treatment targets in rheumatoid arthritis? Low disease activity is sufficiently strict for patients who are anticitrullinated protein antibody negative. J. Rheumatol., 40 (8): 1268-1274.

5. Fadda S, Hamdy A, and Abulkhair E, (2015): Serum levels of osteoprotegerin and RANKL in patients with rheumatoid arthritis and their relation to bone mineral density and disease activity. The Egyptian Rheumatologist, 37: 1-6.

6. Gerolamo B (2012): The role of RANK ligand/ osteoprotegerin in rheumatoid arthritis. Ther Adv Musculoskelet Dis., 4 (4): 225-233.
7. Hudson L and Hay FC (1989): Practical Immunology, 3rd edn. Pbl. Oxford: Blackwell Scientific Publications.

8. Kazim SS, Aysen C, Huseyin D, Emre E, Derya K, Kiymet DE, Aysun SS and Gulden B (2015): Evaluation of various factors leading to osteolytic and antiosteolytic effects in patients with rheumatoid arthritis. Arch Rheumatol., 30 (1): 45-50.

9. Kolahi S, Ghorbanihaghjo A, Rashtchizadeh N, Khabbazi A, Hajialilo M, Noshad H, Boostani F and Mokhtarkhani M (2017): Osteoprotegerin (OPG) levels, total soluble receptor activator of nuclear factor Kappa B ligand (total sRANKL) and RANKL/OPG ratio in patients with rheumatoid arthritis. Rheumatology Research Journal, 2 (1): 23-29.

10. Martinez Sr, Genre F, Mejias RL Ubilla B, Mijares V, Pina T, Corrles A, Blanco R, Martin J, Liorca J, Miguel A and Gay J: (2016): Expression of osteoprotegrin and its ligands RANKL and TRAIL, in rheumatoid arthritis. Scientific Reports, 12: 1-5.

11. Papadaki M, Rinotas V, Violitzi F, Thireou T, Panayoto G, Samiotaki $M$ and Douni $E$ (2019): New insights for RANKL as a proinflammatory modulator in modeled inflammatory arthritis. Front. Immunol., 10: 97-100.

12. Prevoo M, Van THM, Kuper H, Van Leeuwen M, Van Deputte $L$ and Van Riel $P$ (1995): Modified disease activity scores that include twenty-eight joint counts. Development and validation in a prospective longitudinal study of patients with rheumatoid arthritis. Arthritis Rheum., 38 (1): 44-48.

13. Sakae $T$ (2019): RANKL is a therapeutic target of bone destruction in rheumatoid arthritis F 1000 Research, 8 (F 1000 Faculty rev): 533 last update: $17 \mathrm{Jul}$.

14. Sara RM, Fernanda G, Raquel LM, Begona UV, Trinitario P, Alfonso C, Ricardo B, Javier M, Javier $L$ and Miguel AG (2016): Expression of osteoprotegerin and its ligands RANKL and TRAIL in rheumatoid arthritis. Scientific Report, 6: 29713. 
15. Sharp JT, Young DY, Bluhm GB, Brook A, Anne CB, Corbette M, Decker J, Genat HK, GoftenP, Goodman N, Larsin A, Lidsky MD and Pussila $P$ (1985): How many joints in the hands and wrists should be included in a score of radiologic abnormalities used to assess rheumatoid arthritis? Arthritis Rheum. 12: 1326-35.

16. Sousan K, Amir G, Wadereh R, Alireza K, Mehrzad H, Hamid N, Farnaya B and Mchaddesch M (2016): Osteoprotegerin, Total soluble receptor activator of nuclear factor kappa B ligand (Total sRANKL) and OPG/RANKL ratio in patients with rheumatoid arthritis Rheu. Res. J, 1 (2): e7262.

17. Syversen $S$, Goll $G$ and Van der Heijde $D$ (2010): Prediction of radiographic progression in rheumatoid arthritis and the role of antibodies against mutated citrullinated Vimentin: Results from a 10 years prospective study. Annals of the Rheumatic Disease, 69 (2): 345-351.

18. Van Tuyl LHD, Voskuyl AE, Bores M, Geusens P, Landewe RB, Bijkmans BA and Lens WF (2010): Baseline RANKL: OPG ratio and markers of bone and cartilage degradation predict annual radiological progression over 11 years in rheumatoid arthritis. Ann Rheum Dis, 69 (9) 1623.

19. Westergren A (1957): Erythrocyte sedimentation rate range and limitations of the technique. Journal of Medical Science, 3 (1) 20-25.

20. Xu S, Wang Y, Lu J and Xu J (2012): Osteoprotegerin and RANKL in the pathogenesis of rheumatoid arthritis-induced osteoprosis. Rheumatol Int; 32: 3397-403. 
OSTEOPROTEGERIN (OPG) AND SOLUBLE RECEPTOR ACTIVATOR... 1015

\section{تقييم مستوى بروتين الاستيوبروتجرين والر انكل في مرض الروماتويد المفصلي الأئي}

الهام على مطاوع، محمد ابراهيم عارف1، سلامه سعد عبداللطيف1، عبدالشافي احمد حسيب²، هاني محمد حافظ شريف3، د/ احمد محمد سعيد الثوربجي1 1قسم الباثولوجيا الأكلينيكية، 2قسم الروماتيزم والطب الطبيعي، 3قسم الاثعة كلية الطب، جامعة الأزهر

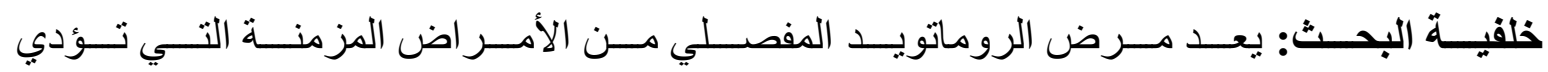

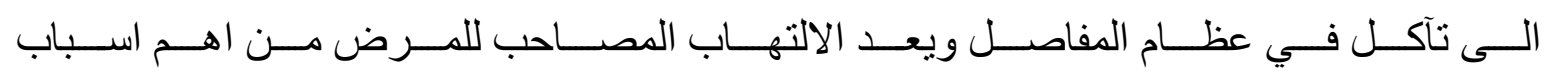

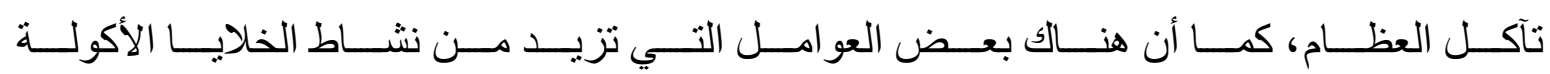
للعظام ومن أهمها مستوى بروتين الاستيوبروتجرين وبروتين الر انكل. بعل

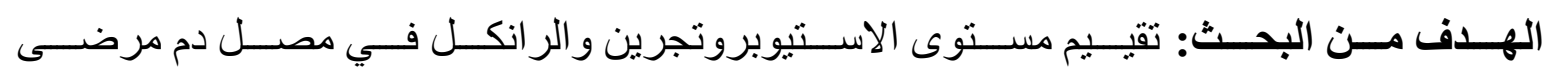

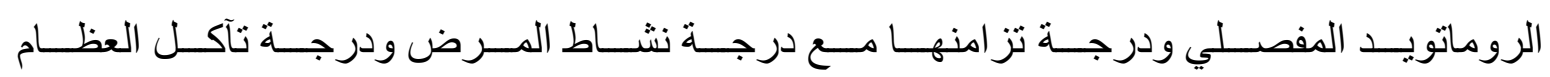
التي تظهر في صور الاشعة.

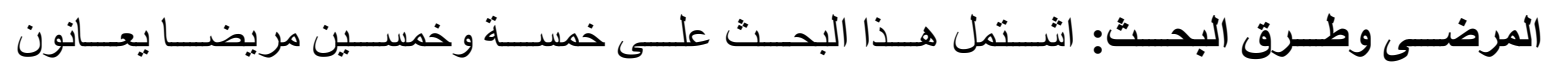

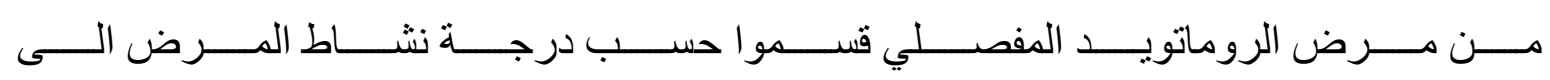

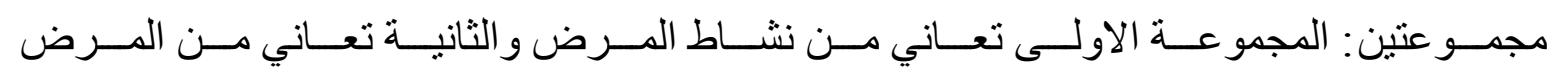

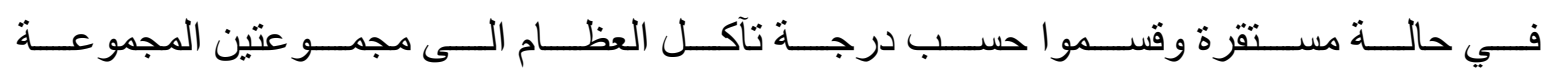

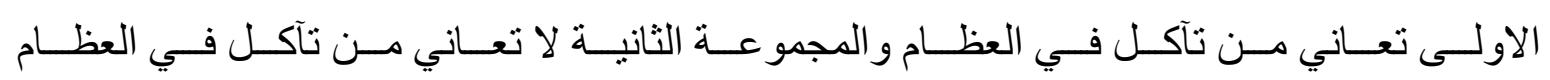

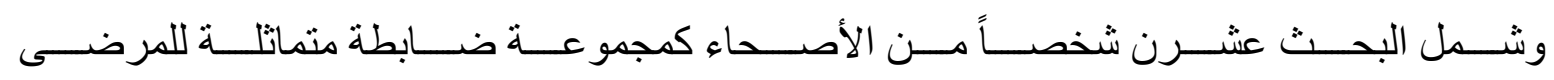

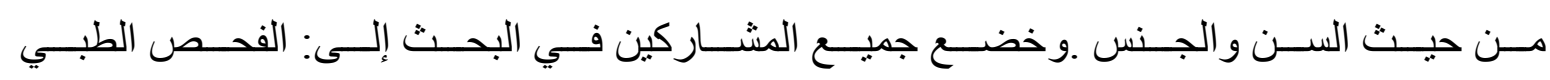

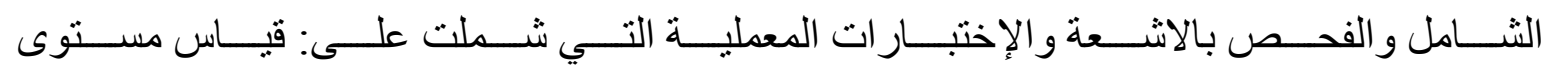

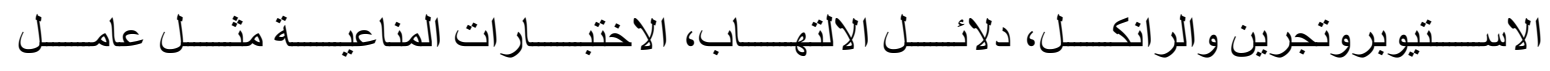

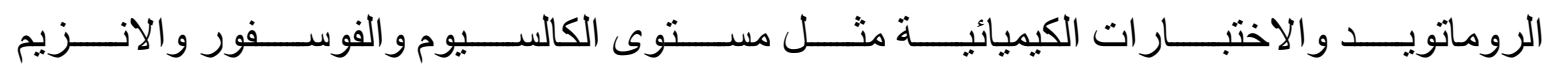
الذي يفرز من العظام، وصورة دم كاملة.

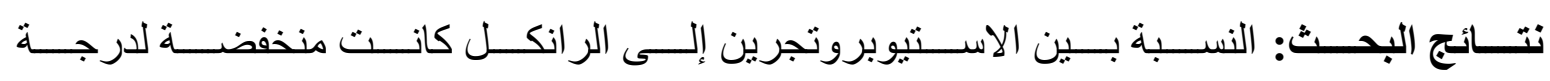

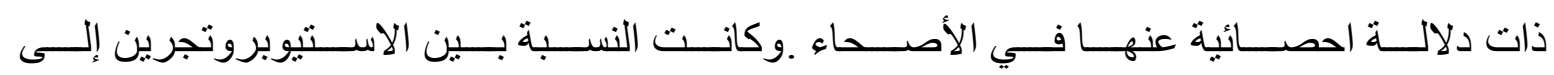




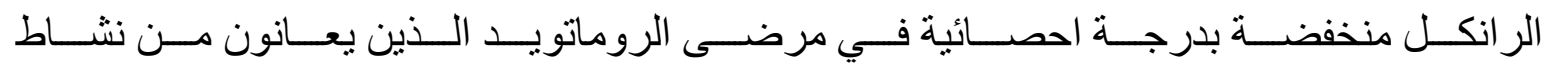

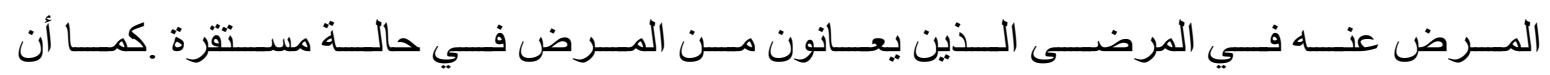

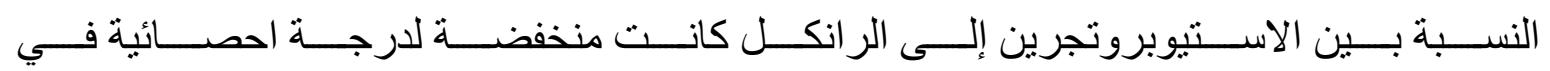

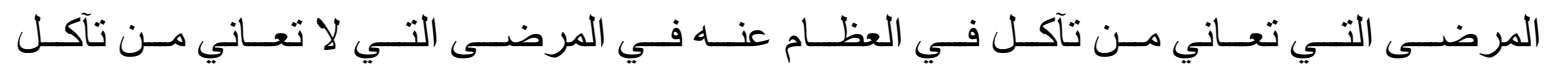

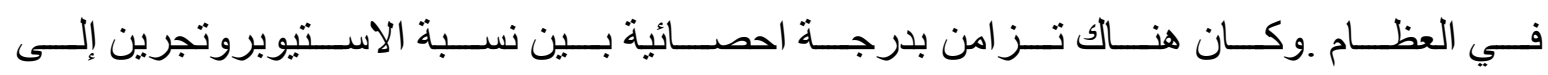

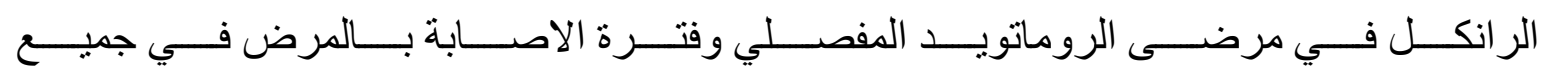

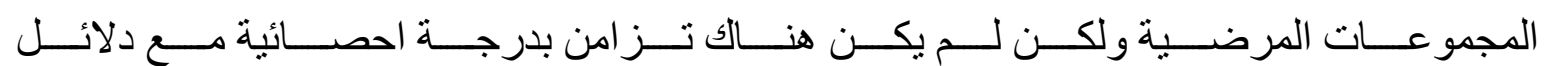

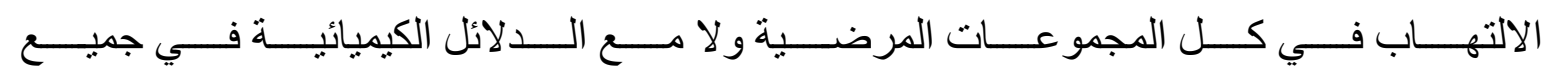

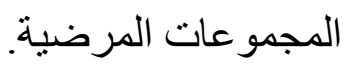

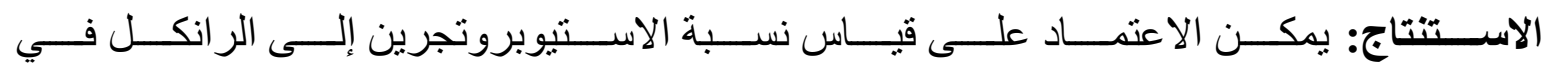

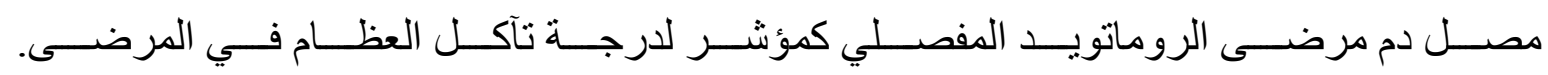

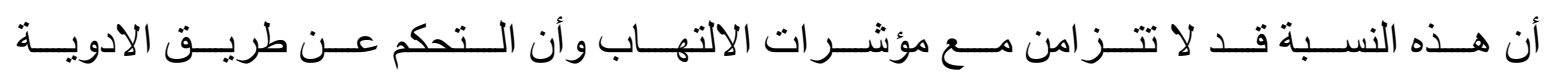

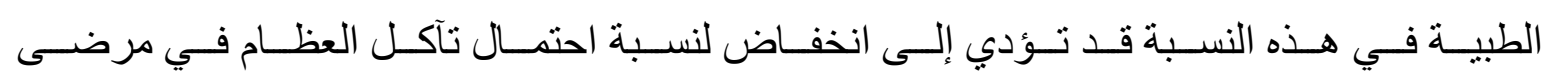
الروماتويد المفصلي. 